\title{
Healthy ageing requires a triple strategy
}

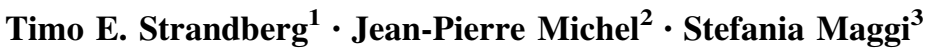

Received: 12 April 2016/Accepted: 22 April 2016/Published online: 6 May 2016

(C) Springer International Publishing Switzerland 2016

Experts often tend to look at things from their own angle, but in this point of view European geriatricians aim to take a global look at healthy ageing, combining views from the United Nations formulated Sustainable Development Goals (SDGs) for 2030 with focus on $<70$ years [1], and ongoing WHO efforts [2], with the focus on older people. We appraise WHO and other experts [3,4] for taking healthy ageing — not only nursing or long-term care-on the foremost arena; it is best for both individuals and society that extra years are spent with good functional capacity and well-being. As geriatricians, we are working for that goal by promoting healthy life styles, targeting prevention and using therapies that are attuned and efficient in our ageing and oldest old patients. Geriatric medicine has been successful; a substantial proportion of current prolongation of life expectancy without cognitive decline $[5,6]$ is certainly explained by improved ageing and old people's care [7]. Whether extra years are also better in quality is a matter of controversy [2].

Moreover, premature mortality and disability are getting more frequent in younger cohorts [8]. Therefore, we must consider healthy ageing in a life course perspective. Few studies exist, but in a recent long-term study from early

The authors are past presidents (TES, JPM) and current president (SM) of the European Union Geriatric Medicine Society (EUGMS).

Stefania Maggi

stefania.maggi@in.cnr.it

1 Department of Geriatrics, University of Helsinki, Helsinki, Finland

2 Geneva Medical School, Geneva University, Geneva, Switzerland

3 Aging Branch, Institute of Neuroscience, CNR, Via Giustiniani, 2, 35128 Padua, Italy midlife to old age, only $5 \%$ of the original cohort of octogenarian men experienced "healthy ageing" (defined by the absence of important non-communicable diseases, intact memory and ADL, happy and socially active) [9]. In older survivors, the proportion has been higher, for example $12 \%$ in a national US sample of $65+$ individuals [10].

However, it is very obvious that the proportion of people with the historical definitions of "healthy ageing" closely hinges on the definitions used [10,11], and it can be argued whether, for example, the absence of adequately treated chronic conditions in old age should be included. In any case, we have still work to do in preventing premature deaths, compressing morbidity and disability to the end of life span, improving function and well-being in later life and favouring dignified death.

Modifiable risk factors should be tackled early, with primordial prevention if possible. As a threat for healthy ageing especially hypertension is important worldwide [12]. High blood pressure is insidious, and strokes and myocardial infarctions are not the only serious outcomes. Equally important from the healthy ageing viewpoint is small vessel dysfunction leading to geriatric syndromes such as cognitive disorders, mobility disability and fall tendency [13], which are prime threats for functional old age. Recently also subclinical atherosclerosis, reflected as increased coronary artery calcium score, was associated with higher risk of dementia in older women [14].

Consequently, to achieve healthy ageing, we need a tripled-edged strategy: (1) promotion of healthy lifestyle at the population level to keep body weight in check, and prevent atherosclerosis and hypertension in the first place: moderate and regular physical exercises, wise and adequate diet, no smoking and low/moderate alcohol consumption, (2) identification and efficient control/treatment of early 
and midlife risk factors, especially hypertension, hypercholesterolaemia, smoking and obesity, to ensure that people have good functional capacity when they enter old age and (3) good coordination of services to treat diseases and prevent frailty and disability of older people, also taking into account their special individual and environmental features. Geriatric medicine is best attuned for the third goal, but geriatricians also need to inform general practitioners and other physicians treating younger people, as well as the health care system overall, how to maximize the odds of healthy ageing and without age discrimination [15], as so well described in the recent first WHO World Report on Ageing and Health [2].

\section{Compliance with ethical standards}

Conflict of interest On behalf of all authors, the corresponding author states that there is no conflict of interest.

Ethical approval This article does not contain any studies with human participants performed by any of the authors.

\section{References}

1. United Nations. Sustainable Development Goals for 2030 https:// sustainabledevelopment.un.org/sdgsproposal.html

2. WHO (2015) World Report on Ageing and Health. http://www. who.int/ageing/events/world-report-2015-launch/en/

3. Bousquet J, Michel JP, Strandberg T et al (2014) The European Innovation Partnership on Active Healthy ageing: the European Geriatric Medicine introduces the EIP on AHA column. Eur Geriatr Med 5:361-362

4. Bousquet J, Kuh D, Bewick M et al (2015) Operative definition of active and healthy ageing (AHA): meeting report. Montpellier October 20-21, 2014. Eur Geriatr Med 6:196-200
5. Jones DS, Greene JA (2016) Is dementia in decline? Historical trends and future trajectories. N Engl J Med 374:507-509

6. Satizabal CL, Beiser AS, Chouraki V et al (2016) Incidence of dementia over three decades in the Framingham Heart Study. N Engl J Med 374:523-532

7. Cruz-Jentoft AJ, Franco A, Sommer P et al (2009) Silver paper: the future of health promotion and preventive actions, basic research, and clinical aspects of age-related disease - a report of the European Summit on Age-Related Disease. Aging Clin Exp Res 21:376-385

8. Martin LG, Schoeni RF (2014) Trends in disability and related chronic conditions among the forty-and-over population: 1997-2010. Disabil Health J 7:S4-S14

9. Rantanen KK, Strandberg TE, Stenholm SS et al (2015) Clinical and laboratory characteristics of active and healthy aging (AHA) in octogenarian men. Aging Clin Exp Res 27:581-587

10. McLaughlin SJ, Connell CM, Heeringa SG et al (2010) Successful aging in the United States: prevalence estimates from a national sample of older adults. J Gerontol B Psychol Sci Soc Sci 65B:216-226

11. McLaughlin SJ, Jette AM, Conell CM (2012) An examination of healthy aging across a conceptual continuum: prevalence estimates, demographic patterns, and validity. J Gerontol A Biol Sci Med Sci 67:783-789

12. GBD 2013 Risk Factors Collaborators (2015) Global, regional, and national comparative risk assessment of 79 behavioural, environmental and occupational, and metabolic risks or clusters of risks in 188 countries, 1990-2013: a systematic analysis for the Global Burden of Disease Study 2013. Lancet 386:2287-2323

13. Strandberg TE, Pitkälä KH, Tilvis RS et al (2013) Geriatric syndromes-vascular disorders? Ann Med 45:265-273

14. Kuller LH, Lopez OL, Mackey RH et al (2016) Subclinical cardiovascular disease and death, dementia, and coronary heart disease in patients 80+ years. J Am Coll Cardiol 67:1013-1022

15. Strandberg T, Pietikäinen S, Maggi S et al (2015) EUGMS. Against age discrimination. Lancet 386:337-338 